\title{
Erratum to: The clinical experience and efficacy of radiofrequency device for wrinkle treatment
}

Yeon A. No ${ }^{1}$ - Beom Joon Kim ${ }^{1}$ - Myeung Nam Kim ${ }^{1}$ • Chang Kwun Hong ${ }^{1}$

Published online: 14 June 2017

(C) Springer-Verlag London Ltd. 2017

Erratum to: Lasers Med Sci

DOI 10.1007/s10103-017-2206-x

The published online version contains mistake in the funding source/grant. The corrected version is shown below.

Funding sources This research was supported by a grant of the Korea Health Technology R\&D Project through the Korea Health Industry Development Institute (KHIDI), funded by the Ministry of Health \& Welfare, Republic of Korea (grant number: HI16C0666).

The online version of the original article can be found at http://dx.doi.org/ 10.1007/s10103-017-2206-X

\section{Beom Joon Kim}

beomjoon@unitel.co.kr

1 Department of Dermatology, Chung-Ang University Hospital, 224-1

Heukseok-dong, Dongjak-ku, Seoul 156-755, South Korea 